\title{
Replik zum Artikel von Macé M. Schuurmans
}

\section{Ulrich Wagner}

Dr. PH, Direktor Nationale Krebsregistrierungsstelle (NKRS)

Das Krebsregistrierungsgesetz (KRG), 2016 von den eidgenössischen Räten verabschiedet, tritt auf den 1. Januar 2020 in Kraft. Im Hinblick auf die Krebserkrankungen regelt das KRG die Erhebung, Registrierung und Auswertung von Daten zu Krebserkrankungen. Ärztinnen und Ärzte, Laboratorien, Spitäler und andere private oder öffentliche Institutionen des Gesundheitswesens, die eine Krebserkrankung diagnostizieren oder behandeln, werden darin verpflichtet, definierte Angaben zu spezifizierten Krebserkrankungen an die kantonalen Krebsregister bzw. das Kinderkrebsregister zu melden. Ärztinnen und Ärzte, welche die Diagnose einer meldepflichtigen Krebserkrankung oder einer möglichen Vorstufe davon feststellen, sind auch dafür verantwortlich, dass Patientinnen und Patienten mündlich und schriftlich über die Krebsregistrierung und deren Zweck sowie über ihr Recht zum Widerspruch informiert werden. Die schriftliche Information wird von der Nationa- len Krebsregistrierungsstelle zur Verfügung gestellt (siehe Begleitschreiben Informationswelle 10/2019 unter www.nkrs.ch).

\section{Angepasster Meldeaufwand}

Die Meldepflicht ist eine bedeutende Prozessveränderung, die auf den ersten Blick bedrohlich wirken kann, denn bisher sammelten die kantonalen Krebsregister die notwendige Information. Sie hat die Vollzähligkeit der Erhebung und den Schutz der Persönlichkeitsrechte von Patientinnen und Patienten als Hauptgründe. Diagnostizierende und behandelnde Ärztinnen und Ärzte können die notwendige Information einfach identifizieren, und sie verfügen schon über sie (Dritte müssen in diesen Prozessteil nicht mehr integriert werden, was die Daten der Patienten schützt). Dieser Schutz wird durch die Wahrnehmung der Informationspflicht ergänzt. 
Weiterhin wird der Meldeaufwand so weit wie möglich reduziert (siehe unten) und einfach gehalten.

\section{Ordentliche Vernehmlassung}

Dem Gesetz und der Verordnung sind ordentliche Vernehmlassungen vorausgegangen. Diese ermöglichten allen betroffenen Organisationen, Interessengruppen sowie einzelnen privaten Personen, sich einzubringen und mit Argumenten zu überzeugen. Die Vertretungen der Ärzteschaft und der Fachverbände waren in jedem der Schritte beteiligt. Die sachliche Richtigkeit, Vollzugstauglichkeit und Akzeptanz von Rückmeldungen aus den Stellungnahmen in den Vernehmlassungen des Gesetzes und der Verordnung wurden genau geprüft, bevor das Gesetz von den Räten beschlossen und die Verordnung durch den Bundesrat erlassen wurde. Die Adressatenliste der Vernehmlassung zur Verordnung samt den eingegangenen Stellungnahmen sind öffentlich. Auch arbeiten die Vertretungen der Meldepflichtigen wie $\mathrm{H}+, \mathrm{FMH}$ und einzelne Fachverbände (z.B. SGMO, SPOG) eng mit anderen Interessengruppen, dem Bund und den Kantonen zusammen, um das neue Gesetz möglichst reibungslos vollziehen zu können. Im Gegensatz zu Forschungsprojekten handelt es sich hier um ein Bundesgesetz, wonach die Angaben zu spezifischen Krebserkrankungen der Meldepflicht unterliegen. Die Patientinnen und Patienten haben das Recht, der Registrierung ihrer Krebserkrankung(en) zu widersprechen.

\section{Vollständige Daten sind unerlässlich}

Damit die im Rahmen der Krebsregistrierung erhobenen Daten einen Beitrag zur Weiterentwicklung, zur Optimierung und zur Steuerung des Gesundheitswesens leisten können, sind die Vollzähligkeit und die Vollständigkeit der Daten zentral. Die Meldepflicht ist dabei eine wichtige Voraussetzung für die Vollzähligkeit der Daten. Die Aufsicht über die Einhaltung der Meldepflicht liegt bei den kantonalen Behörden. Die Vollständigkeit wird von den kantonalen Registern/ dem Kinderkrebsregister u.a. durch Nachfragen bei den Meldepflichtigen sichergestellt. Sie wird von der Nationalen Krebsregistrierungsstelle (NKRS) überwacht. Die NKRS legt die zu erhebenden Variablen fest, definiert sie und standardisiert die Registrierung zugunsten einer optimalen Auswertung.

\section{Vergütung der Leistungen}

Die obligatorische Krankenpflegeversicherung übernimmt nach Artikel 25 Absatz 1 des Bundesgesetzes über die Krankenversicherung (KVG; SR 832.10) die Kosten für Leistungen, die der Diagnose oder Behandlung einer Krankheit und ihrer Folgen dienen.

Der Bund argumentiert nun, dass neben der ärztlichen Leistung an der Patientin oder am Patienten dies auch die damit direkt verknüpften Leistungen sind, wie die Information der Patientin oder des Patienten über die Krebsregistrierung, das Erstellen, das Studium sowie die Weiterleitung von Dokumenten und Berichten an das zuständige Krebsregister, das Benutzen einer Infrastruktur usw. Und zwar unabhängig davon, ob es sich um Berichte in Papierform oder elektronische Dokumente handelt und ob es sich beim Versand um einen Versand per Post oder verschlüsselte E-Mail handelt. Das bedeutet aus Sicht des Bundes, dass die Kosten, die für meldepflichtige Ärztinnen und Ärzte im Rahmen der Meldung von Daten zu Krebserkrankungen anfallen, über die obligatorische Krankenpflegeversicherung bereits abgedeckt sind.

\section{Datenübermittlung geregelt}

Die meldepflichtigen Daten können elektronisch oder in Papierform an das kantonale Krebsregister bzw. an das Kinderkrebsregister übermittelt werden. Um den Meldeaufwand in überschaubaren Grenzen zu halten, dürfen Berichte ans Krebsregister weitergeleitet werden, die im Rahmen der beruflichen Tätigkeit der meldepflichtigen Personen und Institutionen ohnehin erstellt werden. Darunter fallen nach Fachbereich beispielsweise Tumorboard-, Operations-, Pathologie-, Histologie-, Zytologie- oder Spitalaustrittsberichte, Arztbriefe oder Auszüge aus der Krankengeschichte. 2019 fand eine Informationsveranstaltung für die Softwarehersteller der Klinikinformationssysteme (KIS) statt, in welcher sie über das Standard-Austauschformat sowie den Bedarf entsprechender Anpassungen ihrer KIS im Sinne der Arbeitsprozessführung informiert wurden. Die meisten Softwarehersteller arbeiten bereits an den notwendigen Anpassungen ihrer Produkte.

Aussagekräftige und verlässliche Daten zu Krebserkrankungen sind die Voraussetzung, um schweizweit die Entwicklung von Krebserkrankungen über die Jahre hinweg zu beobachten; Präventions- und Früherkennungsmassnahmen erarbeiten und prüfen zu können; die Versorgungs-, Diagnose- und Behandlungsqualität zu evaluieren und die Versorgungsplanung und Forschung unterstützen zu können. Viele Nutzergruppen warten auf die Ergebnisse. So lassen Sie uns mit der Umsetzung des KRG ab 1. Januar 2020 starten.

\section{Literatur}

Weitere Informationen sind unter www.nkrs.ch oder beim BAG zu finden unter folgendem Kurzlink: https://t1p.de/811o 